\title{
THE APPLICATION OF THE THEORY OF SYNTHESIS OF A DELAY LINE WITH A SURFACE ACOUSTIC WAVE FOR A SINGLE-MODE OSCILLATOR OF ELECTRIC SIGNALS IN SOME SENSORS OF NON-ELECTRICAL QUANTITIES
}

\author{
Milan Šimko, Miroslav Gutten, Milan Chupáč, Daniel Korenčiak \\ University of Žilina, Faculty of Electrical Engineering, Univerzitná 1, 01026 Žilina, Slovakia \\ ( $₫$ milan.simko@fel.uniza.sk,+421415132125,miroslav.gutten@fel.uniza.sk,milan.chupac@fel.uniza.sk,daniel.korenciak@fel.uniza.sk)
}

\begin{abstract}
The paper deals with the issue of constructing delay lines on the basis of surface acoustic waves and their application to single-mode oscillators. As a result of a theoretical analysis concrete delay lines are proposed.

In the contribution, there is presented a theory of designing a symmetrical mismatched and matched delay line for a single-mode oscillator of electrical signals on the basis of which there were designed and fabricated acousticelectronic components for sensors of non-electrical quantities.

From the experimental results it can be stated that all of six designed and fabricated delay lines can be effectively used in the construction of single-mode oscillators.
\end{abstract}

Keywords: delay line, oscillator, surface acoustic wave, interdigital transducers.

C 2017 Polish Academy of Sciences. All rights reserved

\section{Introduction}

As selective elements of oscillators of harmonic vibration besides the band-pass filters there are also used delay lines (DLs) and resonators as prospective acoustic-electronic components based on surface acoustic waves (SAW). From a stability point of view we can classify these oscillators as those with volume acoustic waves and LC oscillators. The quality factor of vibrating system of oscillators with SAW is in a range from 100 to 10000 when these oscillators work with the basic harmonic in a frequency range from $10 \mathrm{MHz}$ to $1,5 \mathrm{GHz}$. The main advantages are their small dimensions, low weight, high mechanic strength, low sensitivity to vibrations and the possibility to construct oscillators without using inductors. It increases the demand for their manufacturing and widens the spectrum of their applications in radio electronics, telecommunications and - at the same time - is the incentive to research into the issues connected with their theory and manufacturing.

In the paper, the DLs with SAW for single-mode oscillators are examined and the singlemode oscillation conditions for oscillators are determined and - based on the deduced theory concrete DL are proposed for various applications .

\section{Oscillator with delay line}

The basic principle of operation of an oscillator with a delay line is presented in Fig. 1. A delay line with SAW (3) plugged in the feedback loop of an amplifier (2) is the basic element. A piezoelectric single crystal base (e.g. $Y$ - cut, $Z$ - direction of propagation of $\mathrm{LiNbO}_{3}, \mathrm{ST}, \mathrm{X}$ $-\mathrm{SiO}_{2}$ etc.) on which there are installed the input (IDT 1$)$ and output $\left(\mathrm{IDT}_{2}\right)$ interdigital 
transducers and fabricated with the photolithographic technology. Circuits $(1,3)$ serve to match the DL impedance to the impedance of electronic circuitry.

Because passing a signal from the input to the output of DL is delayed, the examined oscillators are oscillators with a delaying feedback. The theory of these oscillators is well known in the literature $[1,2]$, nevertheless the achieved results concern especially the oscillators with broadband DLs in which the necessary frequency selectivity is secured with additional circuits (e.g. LC), while in a range of frequencies range for possible applications the DL parameters do not differ significantly. A different situation is in the case of DLs with SAW, where DLs themselves have a narrow transmission band and their parameters differ fundamentally in this range. The physical distinctiveness of functioning of the listed components which is related to the phenomena of excitation, extension and reflection of SAW cause that - in addition to providing a delay - the DLs have specific frequency dependencies on the input and output admittances. Their replacement with lumped RLC circuit elements or a broadband DL with an outer selective LC is mentioned only very generally.

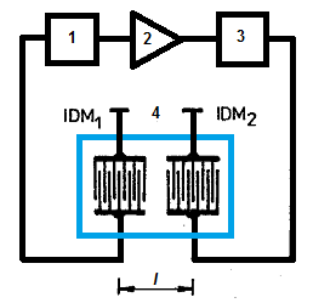

Fig. 1. A block diagram of an oscillator: 1,3-matching circuits; 2 - an amplifier; 4-a delay line with SAW.

It can be considered as an advantage that for the oscillators with SAW, to compensate their static capacitances, compensational inductors plugged in input and output IDTs do not have to be used. By leaving them out, the voltage and current coefficients of transfer radically decrease, which eventually leads to the necessity of increasing the oscillator's active element and thereby to a different action in the process of proposing the DL with SAW.

In the next part we will examine firstly the oscillators without compensation inductors (mismatched DLs with SAW) and then the oscillators with a matched DL.

\subsection{Oscillator with mismatched delay line}

A delay line with SAW used as a selective element of oscillator can be symmetrical or nonsymmetrical. A symmetrical DL is characterized by the same input and output IDTs, whereas a non-symmetrical DL has the input IDT with a small number of electrodes (broadband) and the output IDT with a big number of electrodes (narrowband). In the contribution we will only deal with the symmetrical DL. For simplification we will assume that the input and output admittances of active element, taking into consideration the load, are equal and real, i.e. $g_{1}=g_{2}=g$. The imaginary part of input/output admittance of element (taking into consideration the load) can be included in the static capacity of IDT of DL with SAW.

Solving the complex (1) we determine the parameters of natural oscillations:

$$
K(j \omega)=1 .
$$

The depicted equation can be broken to the equation of phase balance:

$$
\frac{\omega l}{v}+\varphi_{e}=2 \pi k
$$

and the equation of modules' balance: 


$$
K_{0} K_{e}(\omega) K_{1}(\omega) K_{2}(\omega)=1,
$$

where: $K_{0}$ - a coefficient of loop amplification unit consisting of the amplifier and DL; $K_{\mathrm{e}}(\omega)$, $K_{1}(\omega)$ and $K_{2}(\omega)$ - module characteristics of the amplifier, input and output IDTs, respectively; $l=v t_{0}-$ a distance between the centres of input and output IDTsp; $v-$ a velocity of SAW propagation; $t_{0}-$ a delay of the DL; $\varphi_{e}-$ a change of phase of the electric signal in the IDT and the amplifier; $k-$ a positive integer.

An adequate choice of the oscillator active element's amplification enables to attain the phase balance, whereas an appropriate geometrical arrangement of the DL enables to attain the balance of arguments.

For a sufficiently long distance $l$, the value of $\varphi_{e}$ is fundamentally smaller than $\omega l / \mathrm{v}$ and so $\varphi_{e}$ does not need be considered in the first approximation. Then the frequencies at which the oscillations emerge can be determined from (2). Applying the above we obtain:

$$
\omega_{k}=\frac{2 \pi v}{l} k, f_{k}=\frac{k}{t_{0}} .
$$

The indicated frequencies create a discrete spectrum within an interval between single frequencies (Fig. 2b):

$$
\delta f=\frac{1}{t_{0}}
$$

With an appropriate choice of arrangement and IDT geometry, the single-mode oscillation regime can be obtained. In the case of a symmetrical DC, i.e. when the input and output IDTs are equal and have $N$ sections of distance $\lambda_{0}\left(\lambda_{0}=\frac{v}{f_{0}}\right)$, a module characteristic of DL's nearby synchronised frequency can be sufficiently exactly approximated by the function in a form $\frac{\left(\sin ^{2} x\right)}{x^{2}}$, where $x=N \pi \frac{\left(f-f_{0}\right)}{f_{0}}$. Zeros of the module characteristic match the values $x= \pm n \pi$, $(n \neq 0)$. Frequency intervals between zero values are given by the formula:

$$
\Delta f=\frac{f_{0}}{N}=\frac{1}{\tau} .
$$

There can be stated from the presented facts, that the only condition for the single-mode oscillation regime is that all the frequencies of the discrete spectrum (5) need to be identified, with the exception of the synchronous frequency, with zero values of the DL module characteristics: $\delta f=\Delta f$.

Then, from the relations (5) and (6), it results that:

$$
t_{0}=\tau
$$

and - after modifying - the relation:

$$
P_{0}=N
$$

can be obtained, where $P_{0}=\frac{l}{\lambda_{0}}$ and the distance $l$ is expressed as multiples of $\lambda_{0}$. For the singlemode oscillation regime, the DL delay $\left(t_{0}\right)$ and the delay created in IDT $(\tau)$ must be equal (at least approximately), from which it results that the distance between IDT centres must be equal to the IDT length.

It is known that the oscillator's frequency stability is determined by the sharpness of argument characteristic of its resonance system at the working frequency $\omega_{0}$. The sharper this characteristic the higher the frequency stability. 
For the LC oscillators the sharpness of argument characteristic is determined by the time constant $\tau$ of the circuit which is a function of the quality factor $\Theta$ of the resonance system and the working frequency $\omega_{0}$, according to the relation:

$$
S_{f}=\tau_{L C}=\left|-\frac{d \Theta}{d \omega}\right|_{\omega_{0}}=\frac{2 \Theta}{\omega_{0}} .
$$

For the oscillators with DL and SAW there applies:

$$
S_{f}=t_{0}=\frac{l}{v}=\frac{2 \pi P_{0}}{\omega_{0}} .
$$

Applying the previous relations we can introduce a term of the quality factor of the resonance system of oscillator with SAW, as follows:

$$
\Theta=\pi P_{0}=\frac{\pi l}{\lambda_{0}} .
$$

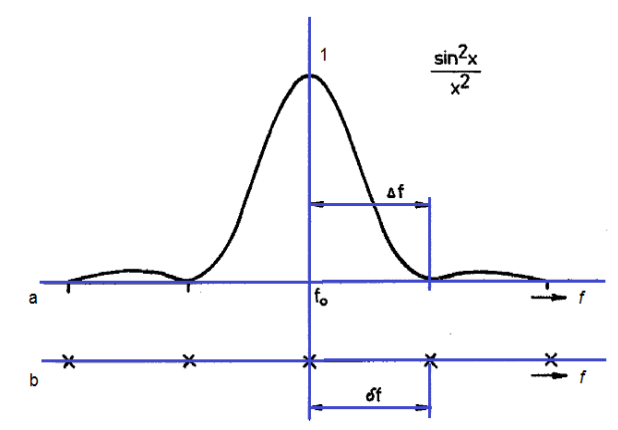

Fig. 2. A module characteristic of IDT (a); the frequency at which the condition of balance of arguments is fulfilled (b).

From (11) it results that the maximum value of quality factor is set by an obtainable value of delay which depends on the propagation velocity of SAW and on the technological possibility to grow mono-crystals, as well as on the operating frequency $\omega_{0}$, which upper limit is given by the capabilities of the photolithographic technology.

A very attractive property of oscillators with SAW is the possibility of independent converting the module and argument characteristics of DL (Fig. 3). Two of IDTs are presented in Fig. 3a, for which the condition (8) is fulfilled and the corresponding module and argument characteristics are shown. A three-fold increase of the delay $t_{0}$ for the same dimensions of IDTs $\left(P_{0}=3 N\right)$ causes a three-fold increase of the phase slope (10), whereas the module characteristic does not change. In the third case (Fig. 3c), the delay and the argument characteristic remain the same as in Fig. 3b, but the length of each of IDT increases three times. It causes narrowing of the DL band-pass in comparison with two previous cases. In the oscillator with a given DL, the oscillations are possible only at one frequency (in DL - Fig. $3 b$ - the condition of the balance of arguments and modules is fulfilled at various frequencies) and this oscillator has a higher frequency stability than the oscillator with a DL shown in Fig. 3a. An increase of frequency stability is achieved by the fact that at an increase of IDT dimensions and thus also at an increase of delay $t_{0}=\frac{l}{v}$ (while $P_{0}=N$ is still valid), the oscillator becomes less sensitive to changes of the electric phase shift (2). 

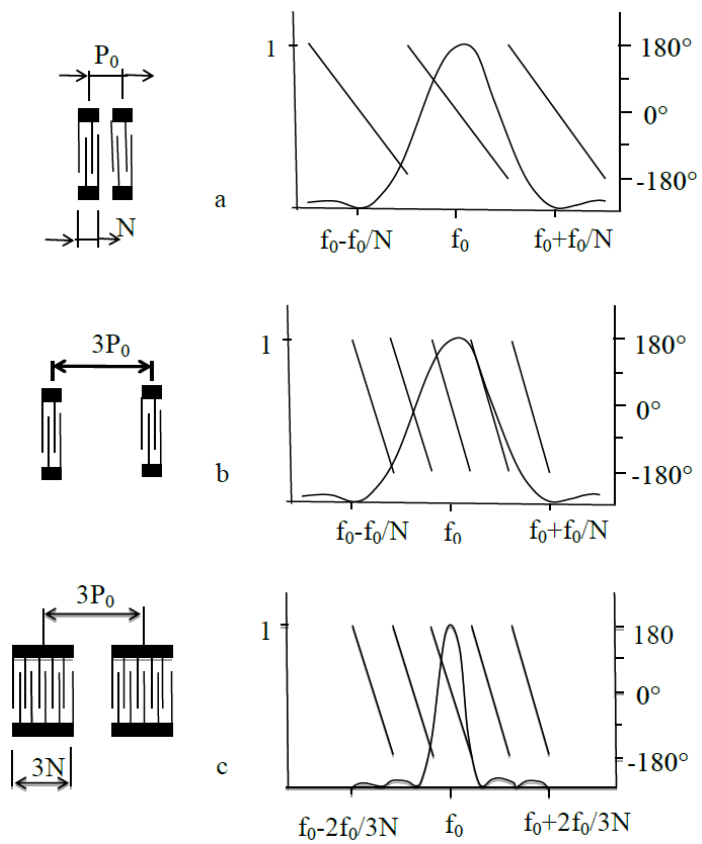

Fig. 3. Module and argument characteristics of IDT of various configurations.

A more accurate single-mode oscillation condition can be determined from the complete (2). Substituting $\varphi_{e}=\psi_{1}-\psi_{2}$ gives $[1,2]$ :

$$
P_{0}=k-\frac{\left(\psi_{1}+\psi_{2}\right)}{2 \pi}
$$

where: $\psi_{1}=\operatorname{arctg} \frac{\left(b_{i}+B_{i}\right)}{\left(g_{i}+G_{i}\right)} ; g_{i}$ and $b_{i}$ are real and imaginary parts of the intrinsic admittance of the $i$ - level gate of linear oscillator's part without the backward coupling of circuit, i.e. without DL with SAW, $G_{i}$ and $B_{i}$ are real and imaginary parts of the input admittance of the $i-$ level gate of DL with SAW nearly short-circuited with the second $(j-$ level) gate $(i=1,2)[3]$.

For piezoelectric materials with a low electromechanical coupling coefficient $K^{2}$ (e.g. $\left.\mathrm{SiO}_{2}\right)$, provided that the components of convertors' admittance are mutually measurable with the components of the input and output admittance of active element and, the following inequality holds:

$$
4 K^{2} N_{i} \frac{\left(1+\chi_{i}\right)}{\pi}<<1
$$

where: $\chi_{i}=\frac{g_{i}}{G_{i}}$ is a matching coefficient, we obtain the following relation to set an optimal value of relative distance between the IDT centres, at which the single-mode oscillation regime emerges at the working frequency $\omega_{0}$. Applying it we obtain:

$$
P_{0}=\frac{2 k-1}{2}+\frac{2 K^{2}}{\pi^{2}}\left[N_{1}\left(1+\chi_{1}\right)+N_{2}\left(1+\chi_{2}\right)\right] \text {. }
$$

It results from (14) that the optimal distance between the IDT centres in DL with SAW approaches an odd number of multiples $\frac{\lambda_{0}}{2}$ at the frequency $\omega_{0}$. 
If the input and output conductances of active element are equal to the corresponding susceptances, i.e. $g_{i}=\omega_{0} C_{T i}$, then:

$$
P_{0}=\frac{4 k-1}{4}+\frac{2 K^{2}}{\pi^{2}}\left(N_{1}+N_{2}\right)
$$

and in the case of a symmetrical DL $\left(N_{1}=N_{2}\right)$ :

$$
P_{0}=\frac{4 k-1}{4}+\frac{4 K^{2}}{\pi^{2}} N
$$

It results from the equation that optimal distance between the IDT centres in this case approaches an odd number of multiples $\frac{\lambda_{0}}{4}$ at the frequency $\omega_{0}$.

\subsection{Oscillator with matched delay line}

To improve the characteristics of oscillators with DL with SAW the matching (compensation) of IDT static capacitance is used. However, the use of compensation circuits has certain advantages and disadvantages [3-5].

Among the advantages are a decrease of inserted damping and an increase of DL transmission coefficient. It enables to achieve a higher output power and to increase effectiveness in comparison with the oscillator with unmatched DL. A disadvantage is the worsening of long-term stability of the oscillator frequency as a consequence of instability of matching circuits. In simple cases we can attain the adjustment by serial connection of the compensation inductor to the IDT clamps (at a low value of the real part of input or output admittance of active element) or by parallel connection (at a high value of the real part). A natural resonance frequency of the created resonance circuit (i.e. serial or parallel) has to be close to the frequency:

$$
\left(\omega_{p}=\frac{1}{\sqrt{L_{k} C_{T}}}\right)
$$

To ensure the single-mode oscillation at $g_{1}=g_{2}=g, \omega C_{T}-\frac{1}{\omega L_{k}}=2 C_{T}\left(\omega-\omega_{p}\right)=g$ for the relative distance between IDT centres, the relation:

$$
P_{0}=k,
$$

where: $k$ - a positive integer, is valid.

From (18) it results the fact that an optimal distance between IDT centres for reaching the single-mode oscillation must be equal to an integer number of $\lambda_{0}$. If the resonance frequency of adapter circuits differs from $\omega_{0}$, then the optimal relative distance between IDT centres can be calculated from $[1,2]$ :

$$
P_{0}=k-\frac{1}{8 K^{2} \omega_{0}}\left[\frac{\omega_{0}-\omega_{p 1}}{N_{1}\left(1+\chi_{1}\right)}+\frac{\omega_{0}-\omega_{p 2}}{N_{2}\left(1+\chi_{2}\right)}\right] .
$$

To increase the output resistance of linear resonance scheme of oscillator (i.e. active element and DL) and also to increase the output signal filtration we can match only the output IDT alone. In this case the optimal distance will be calculated from the relation:

$$
P_{0}=k-\frac{1}{8}+\frac{2 K^{2}}{\pi} N_{1}-\frac{\omega_{0}-\omega_{p 2}}{8 K^{2} N_{2}\left(1+\chi_{2}\right) \omega_{0}} .
$$




\section{Design, discussion and experimental results of symmetrical delay line}

In the paper the DLs with SAW for single mode oscillators are examined. The main achievements of this paper are:

- An initial formulation of a theory of synthetizing a symmetrical DL with SAW. It is the baseline theory according to which there are designed important parts of acoustic-electronic components - the delay lines.

- Verification of the proposed procedure and its accuracy. Based on the theory and the principle of a delay line there were designed and implemented a temperature sensor, a displacement sensor and other sensors for measuring non-electrical quantities.

- A contribution to the state of the art in the field of science concerning sensors of nonelectrical quantities and their general applicability.

- Obtaining the experimental results that confirm effectiveness of all designed and implemented delay lines for the single - mode oscillation applications, and thereby confirm the previously mentioned advantages.

In the paper, the derived theoretical parameters are compared with the experimental ones.

Based on the previous theoretical considerations, a displacement sensor PLO 43 with a symmetrical DL, a thermal sensor PSO 40 with a symmetrical DL and a flow sensor PLO 39 suitable for precise laboratory measurements as well as for normal operating measurements have been designed.

a)

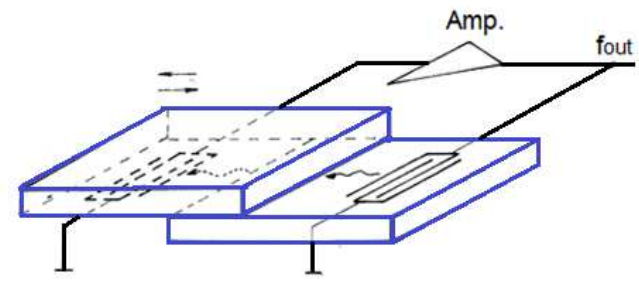

b)

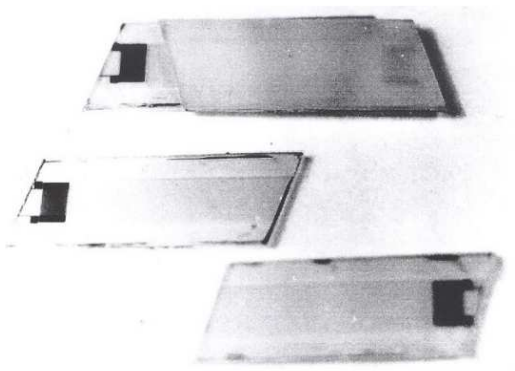

Fig. 4. A structure of DL with SAW (a); a displacement sensor (b).

The displacement sensor consists of two pads with two IDTs installed at the end of propagation path. The plates can move around each other, changing the distance between the IDTs (Fig. 4). On the basis of such constructed a delay line an oscillator with a variable delay in its feedback path is created. The oscillation frequency of the oscillator varies with the change of propagation path length and is given by the relation: 


$$
f=n \frac{1}{\tau_{1}+\tau_{2}},
$$

where: $\tau_{1}=(L-1) / v, \tau_{2}=1 / v ; L-$ a distance between the input and output IDTs; $l-$ a length of overlap of the plates; $v$ - a velocity of propagation of SAW; $v^{\prime}-$ a velocity of propagation of SAW in the overlapping part; $n$ - a number expressing the mode of oscillations.

If the distance between IDTs changes (Fig. 5), with a shift of the plate, from the value of $L$ to $L+\Delta l$, the length of the overlap is reduced to $l-\Delta l$, whereas the oscillation frequency changes from the value $f$ to $f+\Delta f$. In the case when $\Delta l<<L$, we can calculate $(f+\Delta f)$ from the preceding equations.

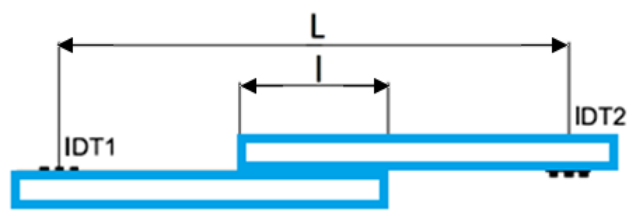

Fig. 5. A displacement sensor geometry.

\section{PLO 43}

Substrate: $Y$ - cut, $Z$ - propagation direction;

$-\mathrm{LiNbiO}_{3}$, thickness of the pad $1 \mathrm{~mm}$.

IDM: $\quad n-101$ electrodes, $l_{e}=l_{0}=10 \mu \mathrm{m}$.
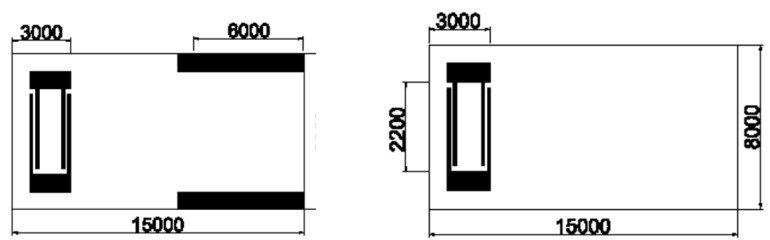

Fig. 6. A schematic of construction (all dimensions in $\mu \mathrm{m}$ ).

Thus,

$$
f+\Delta f \doteq n \frac{v}{L}\left[1-\frac{\Delta l}{L}+\frac{l-\Delta l}{L}\left(1-\frac{v}{v^{\prime}}\right)\right] .
$$

Then, the sensitivity of oscillator frequency variations to changes of an overlap length is given by:

$$
\frac{\Delta f}{\Delta l}=-n \frac{v}{L^{2}}\left[1-\left(\frac{l}{\Delta l}-1\right)\left(1-\frac{v}{v^{\prime}}\right)\right]
$$

and, if $v=v^{\prime}$, then:

$$
\frac{\Delta f}{\Delta l}=-n \frac{v}{L^{2}} .
$$

The number $n$ of modes of oscillations depends on the phase shift that occurs in length, as well as on the frequency-selective properties of IDT. If the plates move from $L$ to $(L+\Delta l)$, the oscillation frequency of the $n$ - th mode moves to the next lower frequency within the reach of the main lobe and the oscillation frequency of the $(n+1)$-th mode moves to the middle of the main lobe (i.e. the frequency of oscillations gradually decreases when the length of overlap decreases; also, there is a discontinuous change of frequency back to a higher frequency). In the 
case where the overlap length increases, the process of the frequency change is reversed. The dependence of the frequency change resulting from the shift is linear.

A schematic of construction of the designed and implemented symmetrical mismatched delay line PLO 43 for a single-mode oscillator is shown in Fig. 6. For its pad there were chosen: $Y$ - cut; $Z$ - direction of propagation of $\mathrm{LiNbO}_{3}$. The substrate thickness is $1 \mathrm{~mm}$. Some of the calculated and measured parameter values are shown in Table 1.

Another delay line, PSO 40, symmetrical and mismatched, was implemented for a temperature sensor (Fig. 7). As a material of the pad a special LST - cut (due to its higher sensitivity), and $X$-direction of propagation of $\mathrm{SiO}_{2}$, have been chosen. The substrate thickness is $0,5 \mathrm{~mm}$. A design scheme is presented in Fig. 8. The fabricated DL with SAW is shown in Fig. 9 and the dependency of inserted damping on frequency - in Fig. 10. Some calculated and measured parameter values are listed in Table 1.

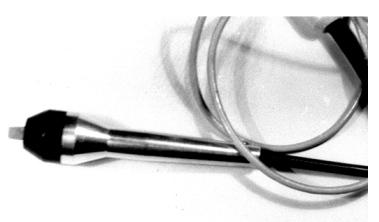

Fig. 7. A fabricated thermal sensor based on SAW.

PSO40

Substrate: $L S T$ - cut, $X$ - propagation direction, $\mathrm{SiO}_{2}$, thickness of the pad 0,5 mm.

IDM: $\quad n-31$ electrodes, $l_{e}=l_{0}=12 \mu \mathrm{m}$.

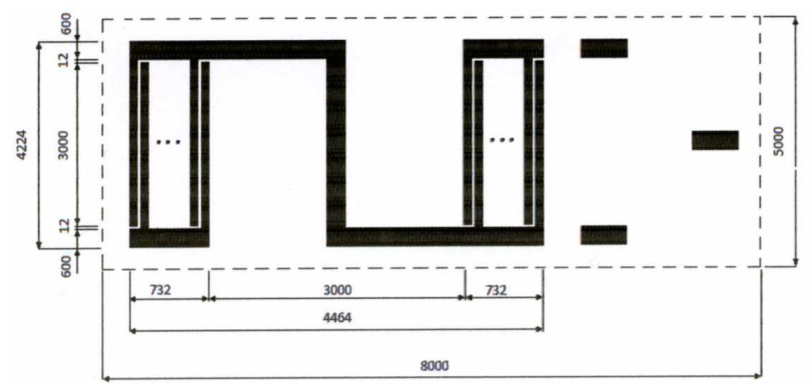

Fig. 8. A design scheme of delay line PSO 40 (all dimensions in $\mu \mathrm{m}$ ).

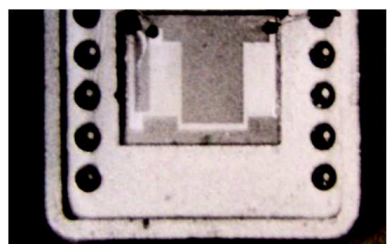

Fig. 9. A photo of fabricated DL PSO 40.

One of the sensors designed on the basis presented in Section 1, was a flow sensor suitable for precise laboratory measurements as well as normal operating measurements. Since the 
components of SAW are extremely sensitive to the temperature change (of the order of $10^{-4} \mathrm{~K}$ ), it has been applied to the construction of the flow sensor. A flow of liquid (or gas) results in a changed temperature of the pad, which is placed on the flowmeter. On this pad, an acousticelectronic component (delay line or resonator) that reacts to the temperature change, is installed.

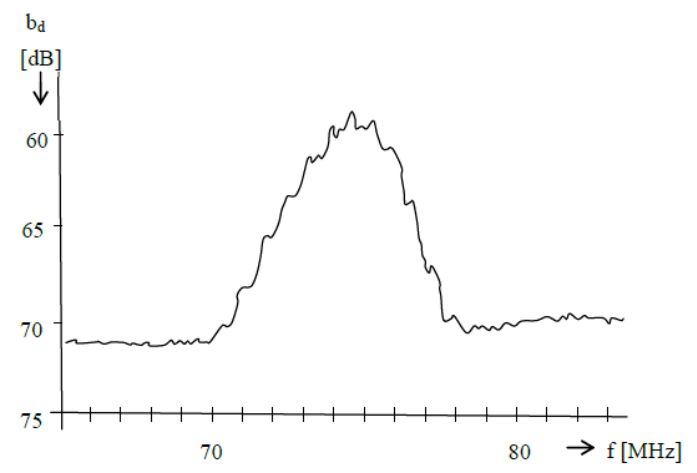

Fig. 10. The dependency of inserted damping on frequency for DL PSO 40.

A block diagram of the flowmeter is presented in Fig. 11.

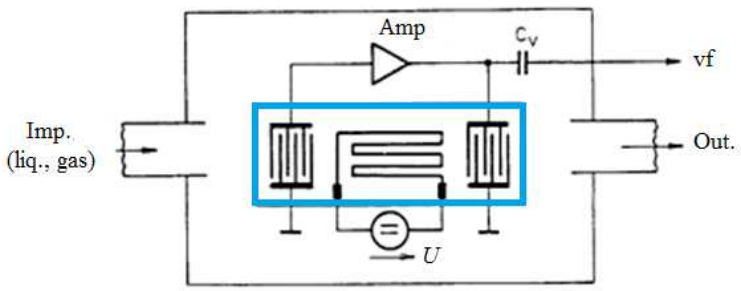

Fig. 11. A block diagram of the flowmeter.

The fabricated flow sensor has been designed on the basis of a delay line PLO $39\left(128^{\circ}\right.$, $Y$ - cut, $X$ - propagation direction of - $\mathrm{LiNbO}_{3}$ with a pad thickness of $1 \mathrm{~mm}$ ). The pad with a DL-based SAW is heated using a thin film heater (meander) of a constant power. The flow of a substance (gas or liquid) (i.e. its flow velocity and the substance properties), results in a change of temperature of pads (the principle of heat transfer by convection). Changing the temperature of the pad causes a linear change of the frequency of oscillator with SAW (in the sensor a delay line has been used, but a resonator can be used instead, too). rule:

The heat transfer by convection per a unit area $A$ can be expressed by the modified Newton's

$$
Q=h A \Delta T \text { or } Q / A=h \Delta T,
$$

where: $h$ - a convective heat transfer coefficient; $A$ - an area; $\Delta T$ - a temperature difference (the convection constant and the temperature difference are determined by the nature of the process).

It is clearly seen from the above relationship that the rate of heat flow grows with increasing the temperature difference and with increasing the transmission coefficient. Increasing the flowing substance speed causes an increase of the transfer coefficient.

The convection process can be expressed as the product of a flowing substance mass $-m$; a specific heat $-c_{p}$ and an average surface temperature gradient $-d t$, as follows: 


$$
Q=m \cdot c_{p} d t
$$

Solving (25) and (26) we can derive a relationship between the flowing quantity and the average surface temperature gradient of acoustic - electronic component with SAW.

Then, the temperature sensitivity of an oscillator with DL-based SAW (we assume that the volume of flowing substance, as already mentioned, causes a change in the temperature of the component with SAW and thus a change of the oscillator frequency) is given by the following equation:

$$
d f=f\left(v_{0}\right)\left[1+a\left(v-v_{0}\right)+b\left(v-v_{0}\right)^{2}+\ldots\right],
$$

where: $a, b$-temperature coefficients of the first and the second order frequency at the reference temperature (zero) $v_{0}$.

A flow characteristic of the implemented SAW-based oscillator (a function of flow vs. frequency) is shown in Fig. 12.

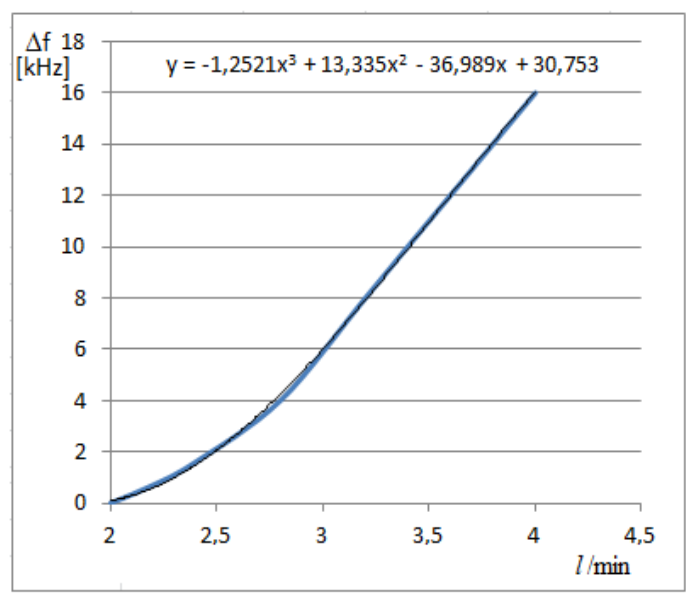

Fig. 12. A flow characteristic of a SAW-based oscillator

As the flowmeter we used a designed, experimentally verified and fabricated symmetrical non-adapted DL - PLO 39 (Fig. 13) with measured and calculated values - Table 1. The dependence of insertion loss on frequency is presented in Fig. 14.

PLO 39

Substrate: $128^{\circ} Y$ - cut, $X$ - propagation direction, $\mathrm{LiNbO}_{3}$, thickness of the pad $1 \mathrm{~mm}$.

IDM: $\quad n=101$ electrodes, $l_{e}=l_{0}=12 \mu \mathrm{m}$.

Meander: meander width $20 \mu \mathrm{m}$.
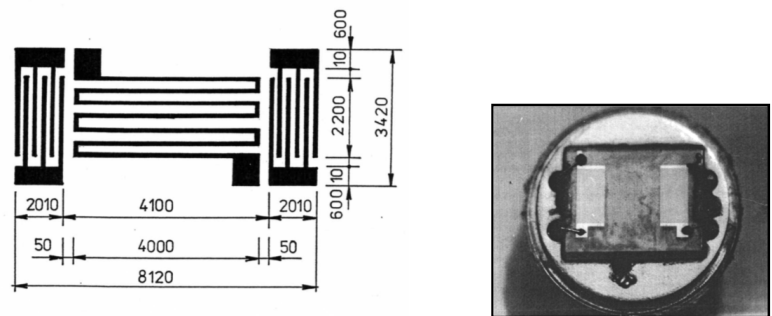

Fig. 13. A photo of the fabricated DL PLO 39 and its design scheme (all dimensions in $\mu \mathrm{m}$ ). 


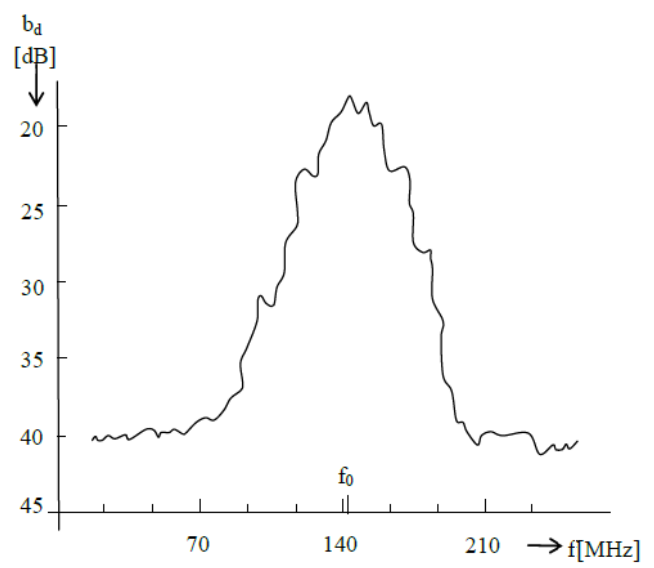

Fig. 14. The dependence of inserted damping on frequency for the DL PLO 39.

A structural design of the flow sensor with SAW is presented in Fig. 15. The cover 2 of the flow sensor is made of fiberglass. The top cover 3 with a seal beneath it (to decrease the influence of external conditions on the piezoelectric substrate with the delay line) is attached with four screws.
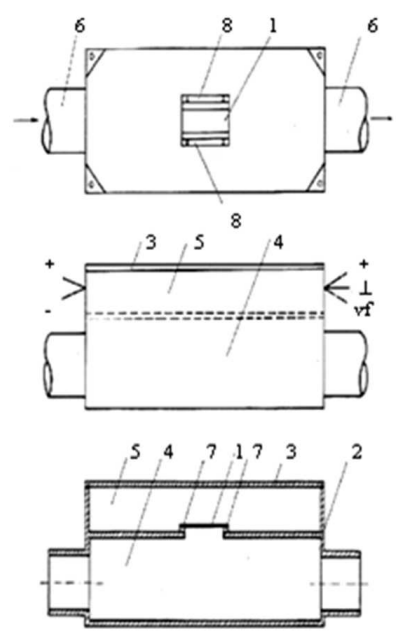

Fig. 15. A design of the flow sensor. 1 - piezoelectric plate with delay line and thin-film heater (meander); 2 - fiberglass cover; 3 - top cover; 4 - flow chamber; 5 - space for electronic part; 6 - inlet and outlet openings; 7 - rubber seal; 8 - holders of piezoelectric pads.

The flow sensor has an inlet and an outlet (6) for the substance flow in and out. The sensor is connected to three wires: DC voltage, RF signal and common ground, implemented by a twowire coaxial cable, and to two wires supplying power to the thin film heater (meander).

The internal space of the sensor is divided into two parts. The bottom part is the flow chamber -4 , through which flows the measured substance. In the top part there is a space for storing the electronic part -5 . In the plate separating the bottom and top parts there is an opening, at the top of which there is fixed a piezoelectric plate with the delay line with a thin film heater (meander) - 1. The temperature of the piezoelectric plate is influenced from the bottom by the flowing substance. The piezoelectric plate is attached with two holders $-8(\mathrm{Z}-$ 
shaped) that are of the same length as the length of the plate. Each holder is attached to a solid plate with two screws. The screws have to be sealed, to prevent contacting the measured substance from the flow chamber with the electronic part. Between the fixed plate and the piezoelectric pad there is a rubber seal - 7 (with a perimeter about that of the piezoelectric pad). A rubber seal is placed also between the piezoelectric plate and the holders. The seals also serve as a thermal insulation preventing a heat exchange between the piezoelectric plate and the fixed plate and holders. Where appropriate, they also work as shock dampers.

In implementing there was used a two-stage amplifier providing a high value of inserted attenuation. For powering the meander there was used a precise stabilized power supply (with a precise control of current and voltage). The flowmeter is attached to a modified evaluation device (a computer with circuits of pre-setting zero and dividing by any number).

Table 1. The parameter values of the fabricated delay line (calculated and measured).

\begin{tabular}{|c|c|c|c|c|}
\hline Parameter & PLO 43 & PSO 40 & PLO 39 & Note \\
\hline vef [m.s-1] & 3483 & 3357 & 3990 & \\
\hline $\mathrm{K} 2$ & 0,0482 & 0,0022 & 0,0482 & \\
\hline $\mathrm{Cs}^{\prime}[\mathrm{pF} \cdot \mathrm{m}-1]$ & 429,3 & 68 & 696 & specific capacity of section \\
\hline$\lambda 0[\mu \mathrm{m}]$ & 60 & 50 & 28 & $\lambda_{0}=v_{e f} / f_{0}$ \\
\hline $\mathrm{le}=10[\mu \mathrm{m}]$ & 10 & 12 & 10 & electrode and gap width \\
\hline foc [MHz] & - & 76,09 & 142,5 & calculated \\
\hline fom [MHz] & - & 76,06 & 142,7 & measured \\
\hline $\mathrm{N}$ & 50 & 15 & 50 & \\
\hline $\mathrm{N}$ & 101 & 31 & 101 & $n=2 N+1$ \\
\hline $\mathrm{I}^{\prime}[\mu \mathrm{m}]$ & 2896 & 732 & 1400 & $I^{\prime}=N \lambda 0$ \\
\hline I $[\mu \mathrm{m}]$ & 7500 & 3732 & 6110 & $\mathrm{I}=\mathrm{P} 0 \lambda 0$ \\
\hline PO & $\approx 130$ & $\approx 83$ & $\approx 220$ & \\
\hline K & 130 & 83 & 220 & \\
\hline CTc [pF] & 47 & 3,06 & 78 & $C T c=C s^{\prime} w N$ \\
\hline CTm [pF] & 45 & 2,62 & 80 & measured \\
\hline Qc & 452 & 261 & 690 & $\mathrm{QC}=\pi \mathrm{P} 0$ \\
\hline Qm & $\approx 439$ & $\approx 260$ & $\approx 695$ & measured \\
\hline $\mathrm{bd}[\mathrm{dB}]$ & 48 & 58 & 18 & inserted damping \\
\hline$w[\mu \mathrm{m}]$ & 2200 & 3000 & 2240 & aperture \\
\hline$\frac{\Delta f}{\Delta l}[\mathrm{~Hz} \cdot \mu \mathrm{m}-1]$ & 300 & - & - & changing the frequency by displacement \\
\hline
\end{tabular}

\section{Conclusion}

In the paper, a theory of designing a symmetrical mismatched and matched delay line for a single-mode oscillator of electrical signals is presented. On the basis of DLs there were designed and fabricated acoustic-electronic components for sensors of non-electrical quantities.

From the experimental results it can be stated that effectiveness of the total of six designed and implemented DLs was confirmed for the single-mode regime. Table 1 shows the calculated 
and measured parameter values of only two sample DLs used in displacement and temperature sensors.

In the further research, the authors will deal with the theory of synthesis of an asymmetrical DL with an interdigital transducer with diluted electrodes.

\section{References}

[1] Neveselý, M. (1986). Akustoelektronika. Bratislava: ALFA.

[2] Dvornikov, A., Ogurcov, V., Utkin, G. (1983). Stabilnyje generatory s filtrami na poverchnostnych akustičeskich volnach, Moskva. Radio a svjaz.

[3] Šimko, M., Chupáč, M. (2012). The theoretical synthesis and design of symmetrical delay line with surface acoustic wave for oscillator with single-mode regime of oscillation. Przeglad Elektrotechniczny, 12.

[4] Kampik, M., Skubis, T. (1998). Ambient temperature coefficient of thermal piezoacoustic sensors operating in a twin configuration. Sensors Actuators, A Physical, 67(1-3).

[5] Kampik, M., Klonz, M., Skubis, T. (1997). Thermal converter with quartz crystal temperature sensor for ACDC transfer. IEEE Trans. Instrum. Meas., 46(2), 387-390.

[6] Šimko, M., Chupáč, M. (2013). Generalisation of transfer function of inter-digital transducer and filter on basic of surface acoustic waves. Przeglad elektrotechniczny, 12.

[7] Chicone, C., Feng, Z.C. (2004). Synchronization phenomena for coupled delay-line oscillators. Physica D., 198, 212-230.

[8] Šimko, M., Chupáč, M., Grega, M., Hronec, R. (2014). The interdigital transducer and ist response in the case of the impact of the inhomogeneous surface acoustic wave. Przeglad elektrotechniczny, 6.

[9] Mamishev, A.V., Sundara-Rajan, K., Yang, F., Yanqing D. (2004). Interdigital sensors and transducers. Proc. of the IEEE.

[10] Glowacz, A., Glowacz, A., Glowacz, Z. (2015). Recognition of monochrome thermal images of synchronous motor with the application of skeletonization and classifier based on words. Archives of metallurgy and meterials, 60(1), 27-32. 